\title{
NEW HIGH ENERGETIC COMPOSITE PROPELLANTS FOR SPACE APPLICATIONS: REFRIGERATED SOLID PROPELLANT
}

\author{
C. Franson, O. Orlandi, C. Perut, G. Fouin, C. Chauveau, \\ I. Gökalp, and M. Calabro
}

\begin{abstract}
Cryogenic solid propellants (CSP) are a new kind of chemical propellants that use frozen products to ensure the mechanical resistance of the grain. The objective is to combine the high performances of liquid propulsion and the simplicity of solid propulsion. The CSP concept has few disadvantages. Storability is limited by the need of permanent cooling between motor loading and firing. It needs insulations that increase the dry mass. It is possible to limit significantly these drawbacks by using a cooling temperature near the ambient one. It will permit not to change the motor materials and to minimize the supplementary dry mass due to insulator. The designation "Refrigerated Solid Propellant" (RPS) is in that case more appropriate as "Cryogenic Solid Propellant." SNPE Matériaux Energétiques is developing new concept of composition with cooling temperature as near the ambient temperature as possible. They are homogeneous and the main ingredients are hydrogen peroxide, polymer and metal or metal hydride, they are called "Hydroxalane ${ }^{\mathrm{TM}}$." This concept allows reaching a high energy level. The expected specific impulse is between 355 and $375 \mathrm{~s}$ against $315 \mathrm{~s}$ for hydroxyl-terminated polybutadiene (HTPB) / ammonium perchlorate (AP) / Al composition. However, the density is lower than for current propellants, between 1377 and $1462 \mathrm{~kg} / \mathrm{m}^{3}$ compared to around $1800 \mathrm{~kg} / \mathrm{m}^{3}$. This is an handicap only for volume-limited application. Works have been carried out at laboratory scale to define the quality of the raw materials and the manufacturing process to realize sample and small grain in a safer manner. To assess the process, a small grain with an internal bore had been realized with a composition based on aluminum and water. This grain had shown very good quality, without any defect, and good bonding properties on the insulator.
\end{abstract}

\section{INTRODUCTION}

Advanced propulsion new energetic material for space application, referred hereafter as RSP, is presented in this paper. The compositions defining this con-

This is an Open Access article distributed under the terms of the Creative Commons Attribution-Noncommercial License 3.0, which permits unrestricted use, distribution, and reproduction in any noncommercial medium, provided the original work is properly cited. 
cept are characterized by a high propulsive performance in terms of specific impulse.

Cryogenic solid propellant, so named because of very low temperature, are widely reviewed in studies carried out by R. Lo et al. (see, for example, $[1,2]$ ). In these works, CSP appears to be a promising new material for propulsion delivering a specific impulse higher than any traditional solid propellant (280-450 s vs. $220-290 \mathrm{~s}[2]$ ). To illustrate CSP potential application, two designs are presented depending on the considered configurations (solid $\mathrm{H}_{2} \mathrm{O}_{2}$ locates between polymer modules or coherent propellant grain). Another interesting result of these studies is a first experimental demonstration of the feasibility of the concept considering $\mathrm{H}_{2} \mathrm{O}_{2}$ /polyethylene (PE) disk stack propellant. Extrapolation to large solid rocket boosters is developed in [3] by considering the case of Ariane 5 booster. It is shown that solid $\mathrm{H}_{2} \mathrm{O}_{2} / \mathrm{PE}$ or solid $\mathrm{O}_{2} / \mathrm{PE}$ can advantageously be compared to $\mathrm{HTPB} / \mathrm{AP} / \mathrm{Al}$ propellant, despite a lower density compensated by an increase in performance.

Refrigerated solid propellants are reviewed and some energetic performances are given. A specific focus is made on the studies performed at SNPE on compositions having a freezing point not too far from the room temperature (i.e., near $0{ }^{\circ} \mathrm{C}$ ). For this study, the presented composition consists in mixing oxidizer and fuel (typically, aluminum powder which size spreads from $200 \mathrm{~nm}$ up to $5 \mu \mathrm{m}$ ). The solid structure is obtained by freezing the material. Safety and feasibility aspects are presented and procedures are defined for producing a refrigerated propellant grain. The last part is devoted to the ballistic characterization of RSP, motor test definition, and experimental results obtained by firing a 600 -gram grain.

\section{THEORETICAL PERFORMANCE CALCULATIONS}

The aim of this preliminary study is to identify potential formulation for RSPs. This study is based on several thermodynamic calculations performed with the SNPE numerical code Ophelie. The composition is selected to reach a solidification point near $0{ }^{\circ} \mathrm{C}$. The considered oxidizers are as follows:

- pure hydrogen peroxide (HP) $\left(\mathrm{H}_{2} \mathrm{O}_{2}\right)$;

- pure water $\left(\mathrm{H}_{2} \mathrm{O}\right)$; and

$-\mathrm{H}_{2} \mathrm{O}_{2} / \mathrm{H}_{2} \mathrm{O}$ mixture.

The fuel materials are:

- polymeric materials (PE or polybutadiene); and

- aluminum. 


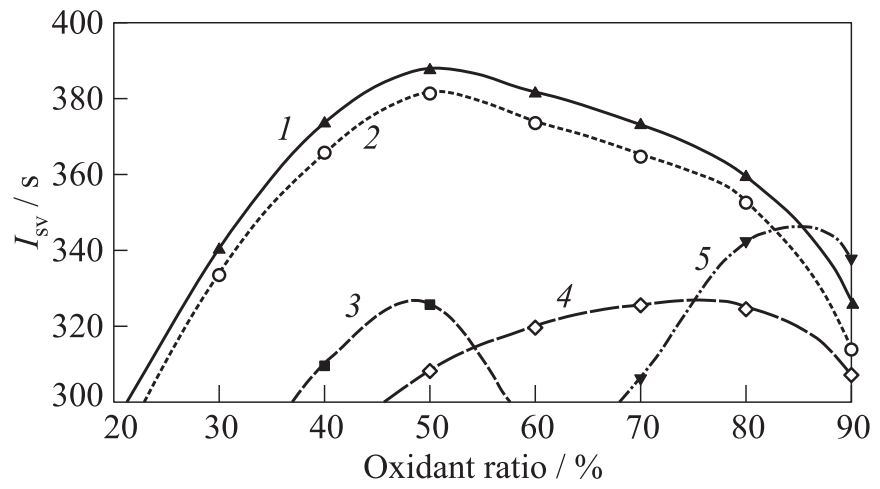

Figure 1 Performance calculations: $1-\mathrm{HP} / \mathrm{AlH}_{3} ; 2-\mathrm{HP}-$ water $/ \mathrm{AlH}_{3} ; 3-$ water $/ \mathrm{AlH}_{3} ; 4-\mathrm{HP} / \mathrm{Al} ; 5-\mathrm{HP} / \mathrm{HTPB}$

The results are presented in Fig. 1. They are obtained by considering a 7-megapascal combustion pressure, vacuum condition, and an expansion ratio of 40. For the purposes of comparison, theoretical specific impulses can be evaluated with regard to $\mathrm{HTPB} / \mathrm{AP} / \mathrm{Al}$ propellant (Butalane ${ }^{\mathrm{R}}$ ) with the delivered specific impulse of $315 \mathrm{~s}$ for a density of $1758 \mathrm{~kg} / \mathrm{m}^{3}$.

Considering the theoretical results shown in Fig. 1, it appears that pure HP is a good candidate due to its solidification temperature at $0{ }^{\circ} \mathrm{C}$ and its large oxidizing properties. Advanced calculations were performed in order to determine the optimized formulation. The higher specific impulse is obtained with aluminum hydride in pure HP (387 s) although the compositions with polymeric material or aluminum as fuel lead to lower values (342 and $327 \mathrm{~s}$, respectively). These values confirm the increase of the performance compared to the $\mathrm{HTPB} / \mathrm{AP} / \mathrm{Al}$ propellant.

Practically, for reducing the two-phase losses, the maximum of specific impulse $(373 \mathrm{~s})$ was found for using a limited quantity of $\mathrm{AlH}_{3}$ with addition of $\mathrm{PE}$ in pure HP. The same calculation performed with aluminum in replacement of $\mathrm{AlH}_{3}$ shows a decrease in the performance which reaches the value of $355 \mathrm{~s}$.

\section{Study Logic}

Pure HP can hardly be handled (due to its very high reactivity) and requires important cares and specific facilities to be produced and stored. Thus, dilute $\mathrm{HP}$ in water was considered. Dilution results in decreasing of the performance. The existence of an eutectic point for this mixture can be considered as an advantage. But the very low temperature of the solidification point can be seen as a limiting factor for potential applications. 


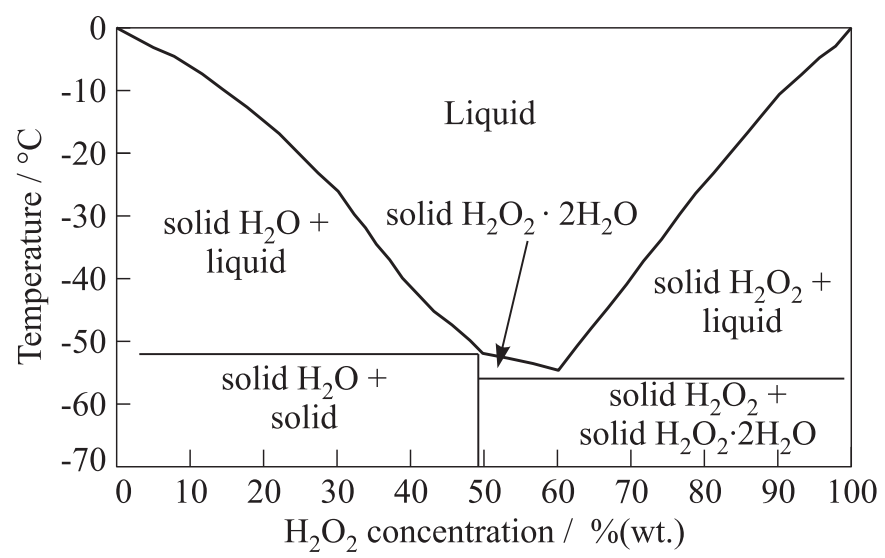

Figure 2 Solid-liquid phase diagram of $\mathrm{H}_{2} \mathrm{O}_{2}$ and $\mathrm{H}_{2} \mathrm{O}$

To obtain a temperature near $0{ }^{\circ} \mathrm{C}$ for a solid propellant, highly concentrated aqueous solution of HP ( $>90 \%)$ is not commercially available; therefore, the use of pure crystal material is not reachable (Fig. 2).

With pure water, performances are slightly decreased and, if $\mathrm{AlH}_{3}$ provides the best result in this case, large formation of solid alumina are foreseen. The presence of that solid phase induces losses due to two-phase phenomena. However, the density observed is relatively low. The presence of aluminum can reduce this effect.

In this way, the first step will consider the use of dilute solution which implies the presence of solid phase dispersed in a liquid solution.

From this first stage, it can be shown that:

- large specific impulses are available with such formulations;

- the best oxidizer is the HP; and

- for safety purposes dilution in pure water is to be considered.

\section{OPERATION AND DEFINITION OF THE FIRING COMPOSITION}

\section{Safer Process}

The aim of this study is to ensure the possibility of ingredients mixing in a safe way. To achieve this goal, the following steps were defined: 
1. Mix two ingredients at room temperature in a porcelain vessel with very small quantities and remote-controlled.

2. Evaluate thermal properties with differential scanning calorimetry (DSC) analysis for a mixture representing the RSP composition.

3. Perform the classical safety tests required for grain making.

This procedure was applied to several components in order to verify their compatibility and optimize the final composition. The main conclusions drawn from this safety study are summarized in the chart (Table 1).

The thermal potential evaluated by DSC is important for a system containing $\mathrm{H}_{2} \mathrm{O}_{2} 30 \%$ with nanoaluminum powder for comparison with the results obtained with a system containing pure water. It can be seen that the energy provided by the exothermal decomposition exhibits peaks which values are higher than $2000 \mathrm{~J} / \mathrm{g}$. Moreover, the temperature of this phenomenon seems compatible with a process at room temperature $\left(T_{\text {onset dec }}>70{ }^{\circ} \mathrm{C}\right)$. This test, used to evaluate thermal properties for a propellant composition, does not allow disqualifying this system.

For this system, no particular sensitivity has been demonstrated with the ISI, ISF, and ES tests.

Additional tests at lower temperature allowed determining the process conditions to formulate homogenous propellant with water or an aqueous solution of HP (30\%). However, composition with water and aluminum is chosen for ballistic characterization and grain realization in order to provide a reference configuration.

\section{Grain Manufacturing}

To obtain a homogenous propellant at low temperature in acceptable chemical compatibility and thermal stability, the manufacturing process was divided into three steps (Fig. 3):

(1) mixing the oxidizing solution with additives;

(2) adding aluminum powder; and

(3) cooling the propellant in the motor configuration to obtain the adequate mechanical properties.

This way one can obtain a cold solid propellant sample with a 30 percent aqueous solution of HP and aluminum powder. For obtaining good solidification, the temperature of the grain storage should be between -35 to $-30{ }^{\circ} \mathrm{C}$. 
Table 1 Results of the safety study

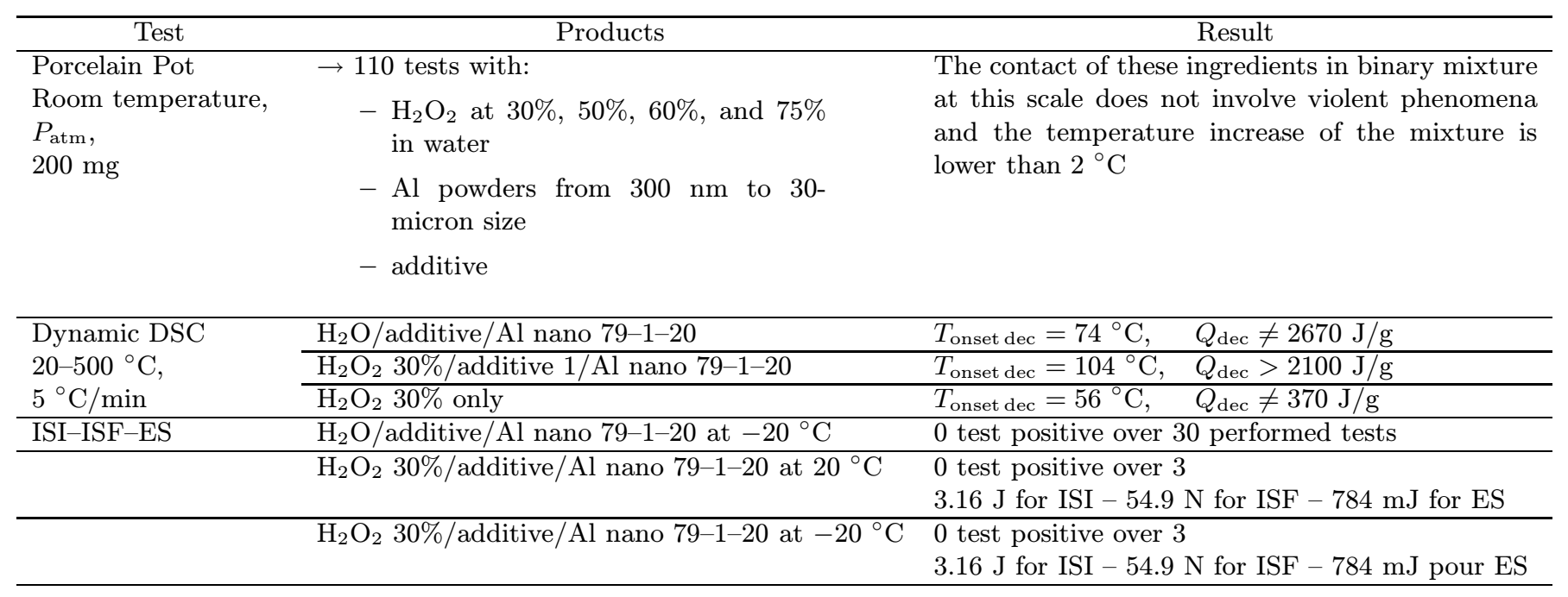



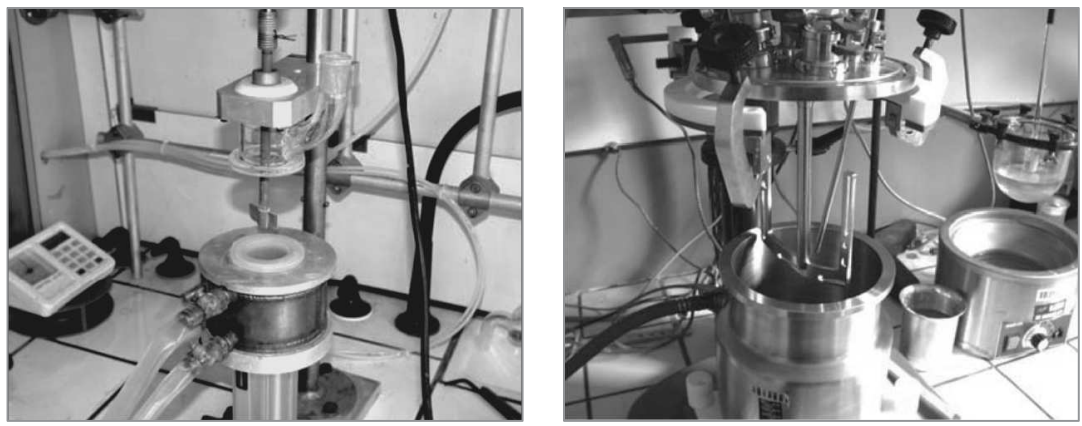

Figure 3 Grain manufacturing

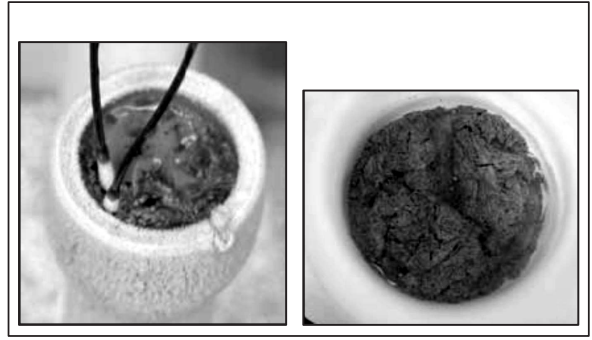

(a)

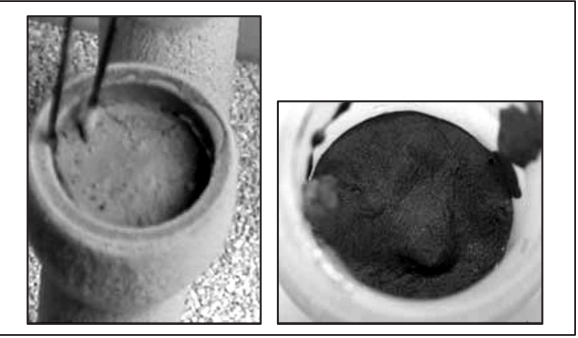

(b)

Figure 4 Refrigerated solid propellant visualization: (a) composition 1: $\mathrm{H}_{2} \mathrm{O}_{2}(30 \%)+$ additive 1 + aluminum (79-1-20); (b) composition 2: $\mathrm{H}_{2} \mathrm{O}_{2}(30 \%)$ + additive $2+$ aluminum (79-1-20)

Several experiments were conducted in order to assess the principal characteristics (stirring speed, temperature of the vessel, amount of aluminum powder, etc.) in experimental conditions compatible with the scaling up.

The formulation had been optimized to obtain the propellant of good quality, without apparent defects such as cracks, at refrigerating the sample of propellant. For example, composition 2 is more homogeneous than composition 1 for which some defects appear at low temperature (Fig. 4).

\section{SMALL-SCALE FIRING TEST}

The proposed formulations are evaluated at small-scale setup in order to measure their burning rate. For a composition close to that presented in this study, a stable end-to-end combustion was observed in [4]. 


\section{Experimental Setup Used for Small-Scale Tests}

One of the main unknowns for such compositions is their burning rate. The data on the burning rate are essential for dimensioning the test motor configuration (i.e., the throat area with regard to the burning surface and burning rate). For these purposes, two types of tests are performed.

The first experimental setup was developed at ICARE* laboratory. It considers a tube filled with RSP which is placed in a closed bomb in order to evaluate the influence of pressure on the burning rate. Special windows designed to support the pressure up to $20 \mathrm{MPa}$ allow visualizing the combustion progression in the tube as it can be seen in Fig. 5. The pressurization is ensured by inert gas such as argon to prevent any postcombustion with hydrogen generated during the test. The RSP mixture is ignited by a hot wire at the top end and the bottom end is closed by a small plane of glass (Fig. 6). These 1-centimeter diameter tubes are made of glass, so the combustion localization can be visualized and recorded with a high-speed camera (Phantom V5 with a speed of 1000 frames/s). The burning rate is otained by derivation of the reactive zone position.

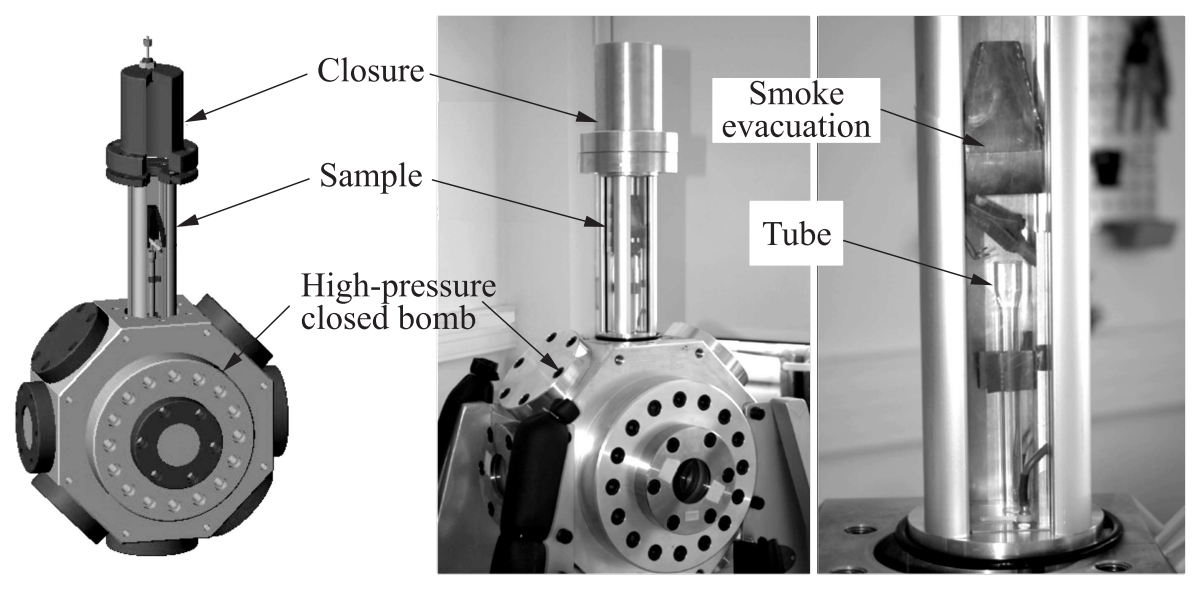

Figure 5 Experimental setup developed at ICARE laboratory (ICARE photo)

The second experimental test is carried out with small BATES grains. The aim of this test setup is to study the ignition and combustion of the RSP in such a configuration. An ignition pellet is placed at the top of the tube and the grain presents a cylindrical geometry with a central bore (Fig. 7). This configuration allows the hot gas generated by the ignition pellet and combustion products to be exhausted. For this test, firings are performed at atmospheric pressure.

*ICARE - Interactions Clouds Aerosols Radiations Etc. 


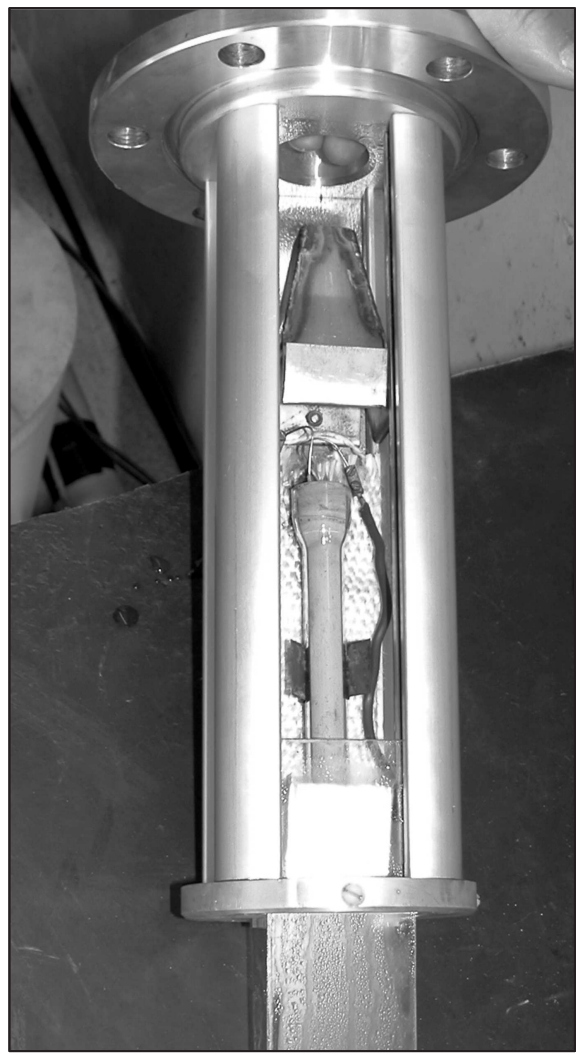

Figure 6 Experimental tube filled with RSP (ICARE photo)

\section{Small-Scale Results}

The results obtained with small scale tests deal with:

- compositions ignition; and

- ballistic data acquisition.

\section{Composition formulations}

Five tested compositions are presented in Table 2 . The compositions vary by the nature of the oxidizer or the size of the aluminum powder. Typically, micrometric particles are $5 \mu \mathrm{m}$ in diameter whereas nanometric aluminum is characterized 


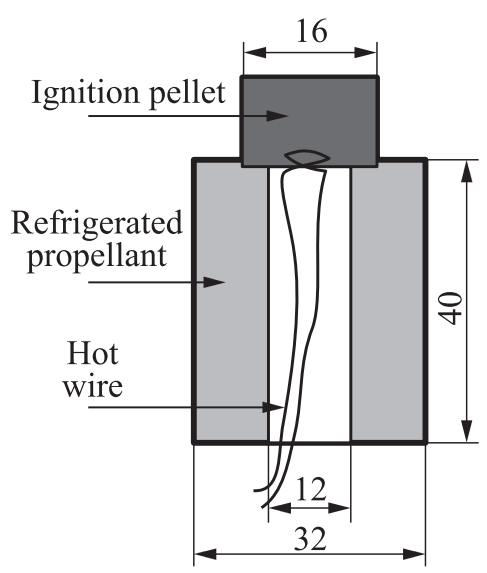

(a)

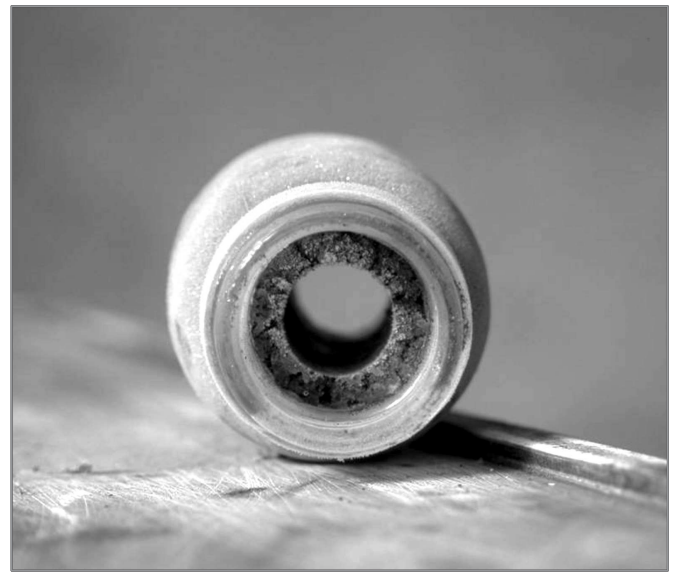

(b)

Figure 7 Schematic view of the mini-BATES setup (a) and RSP material before placing the ignition pellet $(b)$. Dimensions are in millimeters

Table 2 Compositions in small-scale tests

\begin{tabular}{lllccccc}
\hline & & $\# 7$ & $\# 8$ & & $\# 9$ & $\# 10$ & $\# 11$ \\
\hline Oxidizer & $\mathrm{H}_{2} \mathrm{O}_{2}(30 \%)$ & & 70 & & & & \\
\cline { 2 - 8 } & $\mathrm{H}_{2} \mathrm{O}$ & \multirow{2}{*}{70} & & $(10 \mathrm{~cm})$ & $(1 \mathrm{~cm})$ & \multirow{2}{*}{60} & \multirow{2}{*}{60} \\
\hline \multirow{2}{*}{ Al powder } & $\mathrm{Al} 5 \mu \mathrm{m}$ & & 30 & 30 & & 20 & 10 \\
\cline { 2 - 8 } & $\mathrm{Al} \mathrm{nano}$ & 30 & & & 40 & 20 & 30 \\
\hline
\end{tabular}

by an average diameter of $200 \mathrm{~nm}$. The protective layer of $3-5 \mathrm{~nm}$ thickness is composed by alumina without any other specific coating as it is often found for nanometric aluminum powders.

The first two cases (\# 7 and \# 8) are considered to characterize the influence of oxidizer. Considering the stability study results, the 5 -micron powder is used with $\mathrm{H}_{2} \mathrm{O}_{2}$. The \# 9 test tried to optimize the ignition of \# 8 with adding a small amount of nanometric aluminum in contact with the hot wire. A first 1-centimeter thick zone containing nanoaluminum is supposed to be ignited and then to transmit combustion to the composition containing micrometric sized aluminum. The two last cases deal with the compositions with mixed nanometric and micrometric powders. The total amount of aluminum was increased to ensure combustion propagation by increasing the temperature (the stoechiometric ratio is nearly 1:1 proportion). 


\section{Ignition}

In tube tests, the ignition is ensured by a hot wire. The tested compositions presented in Table 2 gave the following results:

- composition \# 7 ignited and completely burned;

- composition \# 8 ignited but the combustion stopped at $1 \mathrm{~cm}$ from the initial surface;

- composition \# 9 was a test to improve ignition. It appears that the combustion transmitting strategy from nanometric to micrometric composition failed: the combustion stopped near the transition region. The lower ignition temperature for nanometric vs. micrometric sized aluminum powders can be a possible explanation; and

- compositions \# 10 and \# 11 are the mixtures of nanometric and micrometric powders. Composition \# 10 exhibited uncontrolled behavior with stops or normal combustion whereas composition \# 11 completely burned.

Mini-BATES grains are ignited by hot gases generated by a propellant pellets. Several ignition compositions were tested to determine the most efficient

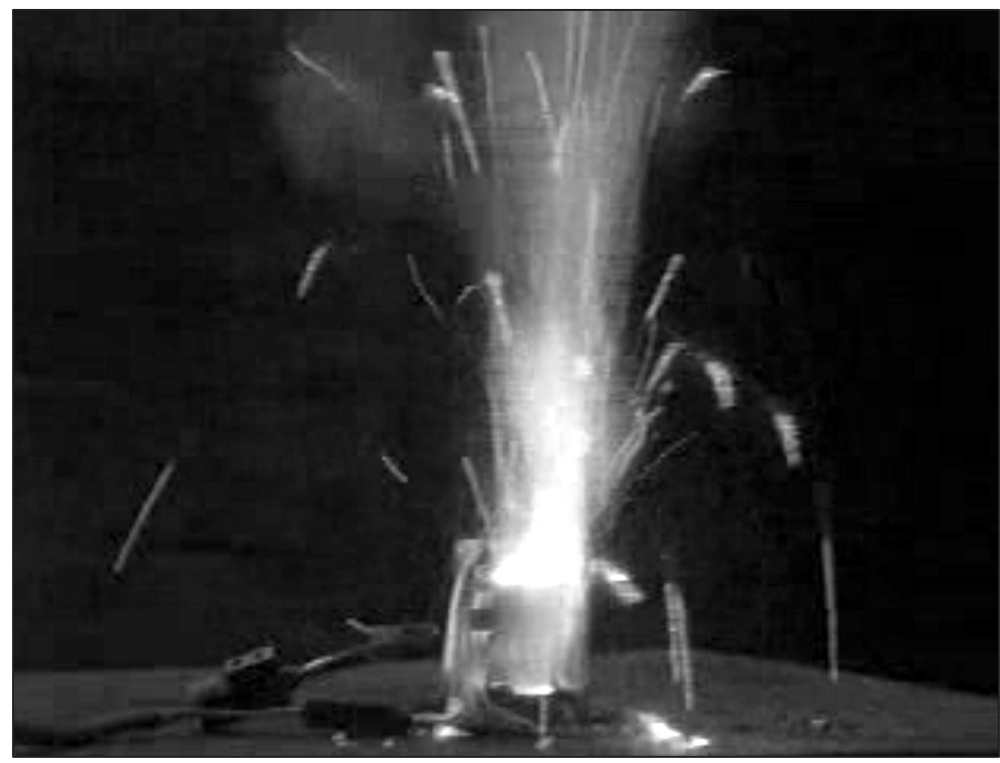

Figure 8 Combustion of mini-BATES grain at atmospheric pressure 
one, but only $\mathrm{HTBP} / \mathrm{AP} / \mathrm{Al}$ propellant successfully provided the ignition of the refrigerated composition. With TGS* and HTPB/AP propellants, a dry material was found in the tube and no combustion was observed. Another interesting result was the time required to ensure ignition followed by complete combustion of the energetic material. It appears that ignition needs quite a long time of $4 \mathrm{~s}$. Figure 8 shows the combustion of a composition similar to \#11. Large residues ejected from the burning surface can be observed, as well as a postcombustion flame resulting from the reaction of generated $\mathrm{H}_{2}$ with oxygen of the ambient air. These results are of practical interest to choose the composition for manufacturing the BATES grain, considering their ability to sustain combustion.

\section{Ballistics}

In tests with complete combustion, the burning rates were measured and are presented in Table 3. For modeling the burning rate, a conventional Vieille law $r_{b}=a P^{n}$ is used $\left(r_{b}\right.$ in $\mathrm{mm} / \mathrm{s}$ and $P$ in $\left.\mathrm{MPa}\right)$.

Table 3 Ballistic results for different tested compositions

\begin{tabular}{lll}
\hline \multicolumn{1}{c}{ Composition } & $a$ & \multicolumn{1}{c}{$n$} \\
\hline $\mathrm{H}_{2} \mathrm{O} /$ Nano $(60 / 40)$ & 1.57 & 0.36 \\
$\# 10 \mathrm{H}_{2} \mathrm{O} /$ Nano/Micro $(60 / 20 / 20)$ & 0.87 & 0.12 \\
$\# 11 \mathrm{H}_{2} \mathrm{O} /$ Nano/Micro $(60 / 30 / 10)$ & 3.08 & 0.3 \\
\hline
\end{tabular}

\section{BATES FIRING}

Test firing of a grain was considered as a challenge. It was not obvious that the behavior observed at small scale should be the same as at a larger scale. It has to be noted that this section aims to demonstrate the validity of the procedure to obtain and fire such a grain.

The manufacturing procedure was adapted to the specific properties of the material, especially to take into account thermal expansion occurring during the freezing phase. The manufacturing of an inert grain (aluminum was replaced by silica particles) confirmed that the thermal insulation was not damaged by RSP expansion. Moreover, this grain was stored for several months in a refrigerated room. No change in the geometry was observed and the RSP adhesion to the insulation material seemed to remain excellent.

\footnotetext{
${ }^{*}$ TGS — triglycine sulfate.
} 


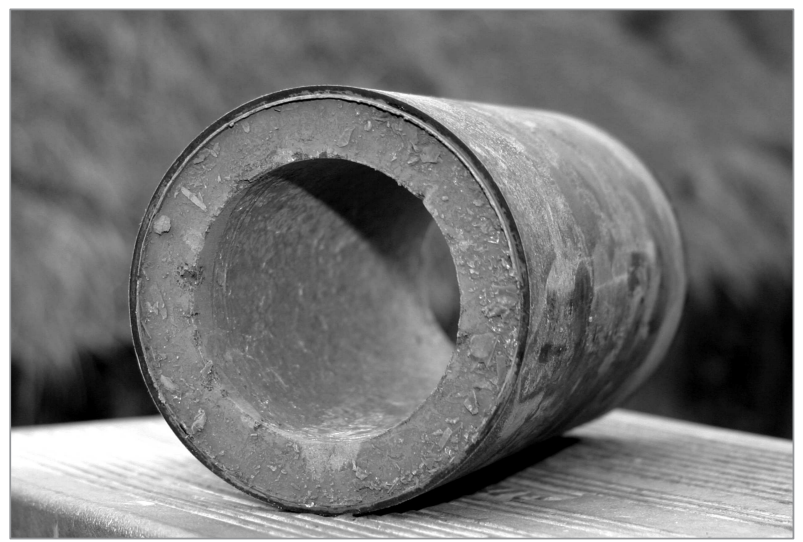

Figure 9 BATES grain after storage

Two axisymmetrical RSP grains were manufactured following the procedure and the formulation recommendations given in Section 2. The visual quality of grains was satisfactory, without any particular defect.

Cylindrical BATES and half-BATES grains were fired at $\mathrm{CRB}^{*}-\mathrm{SNPE}$ Matériaux Energétiques Rechearch Center at the end of 2006. The dimensions for the BATES configuration were as follows: outer diameter of $86 \mathrm{~mm}$, inner diameter of $60 \mathrm{~mm}$ for a total length of $157 \mathrm{~mm}$. Figure 9 shows a photograph of the grain with the total mass of $550 \mathrm{~g}$ ( $220 \mathrm{~g}$ for the half-BATES). They were made of the \# 11 composition $\left(\mathrm{H}_{2} \mathrm{O}\right.$ and a mixture of micrometric/nanometric aluminum powders) and were stored at $-30{ }^{\circ} \mathrm{C}$ during one day before firing. The grains did not have any defect, such as crack. During this period, no deformation of the geometry (i.e., no thermal expansion) was measured before introducing in the test motor (see Fig. 9). With regard to ballistic results, the nozzle throat was dimensioned to obtain combustion at 2 and $3 \mathrm{MPa}$ for the half-BATES grain and BATES grain, respectively. The ignition was facilitated by a HTPB/AP/Al propellant chosen due to high temperature of the provided gases and its ability to ignite such a composition. The web thickness of the igniter was dimensioned for a 4-second burning. Its geometry is a ring inhibited on its lateral surfaces. The 4-second time was considered to be long enough to allow the ignition of the refrigerated composition at BATES scale.

Figure 10 shows a visualization of the plume resulting from the RSP combustion. The presented time moment corresponds to few milliseconds after the end of combustion of the ignition block. At this time, the RSP burns by its own as it was observed during small-scale test. The very luminous flame is generated

${ }^{*}$ CRB - Centre de Recherches du Bouchet. 


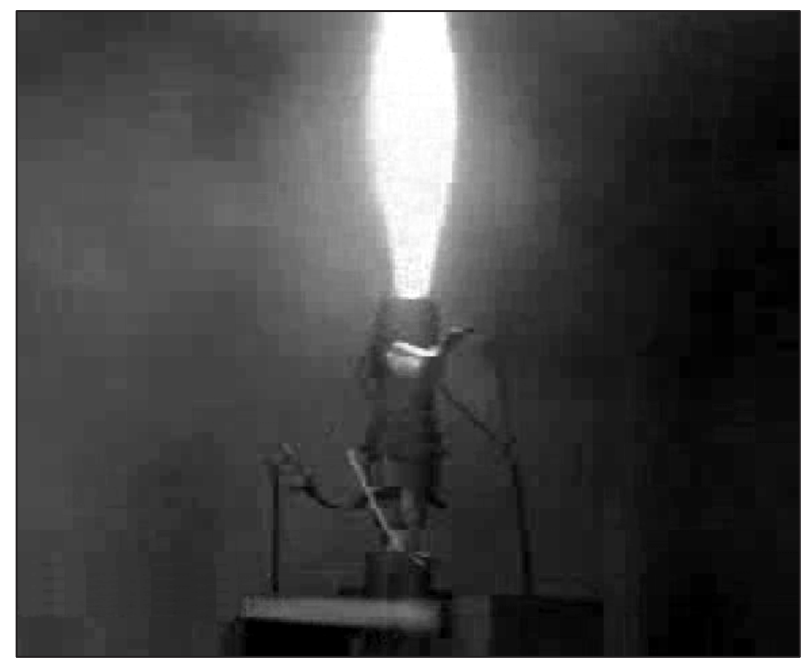

Figure 10 Firing visualization

by afterburning of hydrogen released by the decomposition of the RSP in the ambient air oxygen.

The combustion chamber was equipped with pressure transducers. The pressure records correspond to the following firings:

- igniter alone;

- half-BATES grain; and

- BATES grain.

Considering the igniter, its firing time was observed to correspond to the desired value of $4 \mathrm{~s}$ and the measured pressure maximum was $1.6 \mathrm{MPa}$. The BATES and half-BATES pressure curves indicate that the grain ignition occurred at the beginning of firing. For this reason, an important ignition peak was observed and the higher pressure evolution reduced the firing time of the igniter. It can be deduced from the analysis of this stage that the grain ignition is not homogenous on the whole surface. The BATES surface available to hot gases was twice as that of the half-BATES grain. With the hypothesis of homogenous ignition, these two configurations cannot exhibit the same pressure levels as it was measured for more than $1 \mathrm{~s}$. This phenomenon is also confirmed by the very long end of combustion. Nevertheless, regular combustion was observed after this ignition phase.

A considerable amount of solid residue was found in the combustion chambers in both half-BATES and BATES firings. Performed analyses revealed that a 
small portion of unburned aluminum is present in these residues. The mass fraction (around $13 \%$ of the residue) allows an estimation of $17 \%$ of initial aluminum not participating in combustion and as a consequence, in the performance. This result may explain the lower measured pressure (2 MPa vs. $3 \mathrm{MPa}$ expected for BATES configuration).

\section{CONCLUDING REMARKS}

The RSP concept offers the possibility to significantly increase the energy performance levels of solid propellants.

SNPE Matériaux Energ" etiques is developing new formulations called "Hydroxalane ${ }^{\mathrm{TM}}$ " with a storage temperature as near as possible at the room temperature (the range 0 to $-20{ }^{\circ} \mathrm{C}$ seems accessible) to minimize the drawback induced by the need for grain cooling. These compositions are homogeneous and the main ingredients are HP, polymer, and metal or metal hydride. The expected specific impulse is between 355 and $375 \mathrm{~s}$ against $315 \mathrm{~s}$ for the HTPB/AP/Al composition. However, the density is lower than that of modern propellants, between 1377 and $1462 \mathrm{~kg} / \mathrm{m}^{3}$ compared to around $1800 \mathrm{~kg} / \mathrm{m}^{3}$. This is a disadvantage for volume-limited applications.

The manufacturing process of the propellants and the selection of raw materials exhibiting good chemical compatibility with HP had been assessed by laboratory tests. To assess the manufacturing process, a grain (550 g) with an internal bore was prepared with a composition based on aluminum and water. This grain has shown very good quality, without any defect, and good bonding properties with the insulator. The combustion properties of this composition has been assessed by firing small grains $(\varnothing=32 \mathrm{~mm}, H=40 \mathrm{~mm})$. When one part of the aluminum was nanosized, the grains burned well with a low-pressure exponent.

\section{ACKNOWLEDGMENTS}

This study was supported by the Centre National d'Etudes Spatiales (CNES). The authors would like to thank CNES for support and fruitful discussions.

\section{REFERENCES}

1. Lo, R.E. 1998. A novel kind of solid rocket propellant. Aerospace Sci. Technol. $2(6): 359-67$. 
2. Adirim, H., R.E. Lo, N. Piltz, H. Schöyer, S. Gläser, V. Weisser, H. Meusemann, and F. Caramelli. 2005. Cryogenic solid propulsion technology development status. AIAA Paper No. 2005-4093.

3. Piltz, N., H. Adirim, and R. Lo. 2005. Investigation of cryogenic solid propulsion application for large solid rocket booster. IAC-05-D2.5.04.

4. Risha, G. A., S. F. Son, R. A. Yetter, V. Yang, and B. C. Tappan. 2007. Combustion of nano-aluminum and liquid water. Proc. Combust. Inst. 31(2):2029-36. 